\title{
Fabrication of a Novel Polymer Nanohybrid Electrode Material PANI-BaMnO 3 for High Power Supercapacitor Application
}

\author{
M. Shanmugavadivel, V.Violet Dhayabaran* and M. Subramanian \\ Department of Chemistry, Bishop Heber College, Tiruchirapalli-620 017, India
}

Received October 4, 2016; accepted February 20, 2017

\begin{abstract}
The electro active inorganic-organic nanohybrid material Polyaniline- $\mathrm{BaMnO}_{3}$ has been synthesized by the surfactant assisted chemical polymerization reaction of aniline with nanocrystalline $\mathrm{BaMnO}_{3}$. Electrochemical studies have been performed using cyclic voltammetry and galvanostatic charge-discharge measurements. The enhanced electrode performance originates from the synergistic effect of PANI and $\mathrm{BaMnO}_{3}$. The hybrid delivers very high specific capacitance of $560.5 \mathrm{Fg}^{-1}$, energy density of 32.01 whkg ${ }^{-1}$ and power density of $400 \mathrm{wkg}^{-1}$, respectively. The hybrid also exhibits very high stability and excellent cycling performance with less than $5 \%$ capacity loss over 500 cycles.
\end{abstract}

Keywords: Organic-inorganic nanohybrid, surfactant assisted chemical polymerization, charge-discharge, supercapacitor.

\section{Introduction}

Numerous electroactive materials such as transition metal oxides and conducting polymers have been extensively investigated to enhance the capacitance and energy characteristics of supercapacitors. Manganese based binary and ternary metal oxide nanomaterials $\left(\mathrm{MMnO}_{\mathrm{x}}\right)$ have been used as cathode materials for supercapacitors to improve their energy and power densities [1-3]. Nanocrystalline metal oxides usually possess the characteristics of large surface area, high conductivity, electrochemical stability and pseudo-capacitive behaviour. Another promising electrode material for supercapacitor application is conducting polymers. Because of their unique properties such as high specific capacitance, involvement of whole polymer mass in the charging process, high conductivity in the doped state and fast charge/discharge electron transfer kinetics, many researchers have been involved in the development of supercapacitors using various conducting polymer based electrode materials [4-

\footnotetext{
* Corresponding author. E-mail address: violetstaff@yahoo.co.in
} 
9]. Among the conducting polymers, polyaniline has been of much interest for supercapacitor application, due to its environmental stability and high electrical conductivity [10-13]. The electrochemical activity of the dedoped form of PANI is very low because of the volumetric shrinkage during the elimination of counter ions, and the low conductivity of this state leads to high ohmic polarization, which reduces the reversibility and stability of the supercapacitor. To overcome these problems, an organic polymer-metal oxide nanohybrid material has been chosen as an electrode material for the supercapacitor. The integration of a nanostructured transition metal oxide into a conducting polymer matrix provides an effective way to harness the electrochemical activity of nanosized oxide clusters. By anchoring the transition metal oxides into conducting polymers, the reversible redox chemistry of the soluble polyoxometalate clusters can be combined with that of the conducting polymer to facilitate energy storage applications [14-19]. The results of a recent study reported that nano sized $\mathrm{BaMnO}_{3}$ is an excellent ceramic material which has high specific capacitance value and high rate of discharge [20]. The present work describes the synthesis and electrochemical performance of $\mathrm{PANI}-\mathrm{BaMnO}_{3}$ nanohybrid material. The nanocrystalline $\mathrm{BaMnO}_{3}$ is firmly fixed throughout the PANI matrix, which improves the crystallinity, thus increasing the stability of the polymer. $\mathrm{BaMnO}_{3}$ and the polymer chains attract each other, and the synergistic effect results in greater electrochemical performance compared to the total performance of the individual components working separately. The electrochemical tests demonstrate that PANI-BaMnO 3 is a promising electrode material for the use of supercapacitors.

\section{Experimental}

The PANI-BaMnO 3 nanohybrid was synthesized by the surfactant assisted dilute polymerization method. Based on our earlier work, large amounts of nanocrystalline $\mathrm{BaMnO}_{3}$ were synthesized by the solution combustion method, and this has been reported elsewhere [20]. Aniline was purified by distillation under reduced pressure. $0.2 \mathrm{M}$ aniline was dissolved in $200 \mathrm{~mL}$ of $1 \mathrm{~N} \mathrm{HCl}$. The prepared $\mathrm{BaMnO}_{3}(5 \mathrm{wt} \%)$ was suspended in this solution and mechanically stirred for $30 \mathrm{~min}$. Dodecylbenzene sulphonic acid (DBSA) and Triton X-100 (2:1 mole ratio) were dispersed into this reaction mixture and continuously stirred. $1 \mathrm{M} \mathrm{HCl}$ solution $(30 \mathrm{~mL})$ containing ammonium persulfate was added dropwise to the monomer-surfactant solution under stirred condition. The addition of the oxidizing agent was very slow and the whole process was carried out in an ice cold condition $\left(0-5{ }^{\circ} \mathrm{C}\right)$. After the addition of the oxidizing agent, stirring was continued for 6 hours to ensure completion of the polymerization reaction. The precipitated polymer hybrid was filtered and washed with distilled water until the filtrate was colourless. Finally, the polymer was thoroughly washed with an aqueous solution of $\mathrm{HCl}$. The resulting green powder was rinsed with methyl alcohol, filtered and dried under dynamic vacuum at $80{ }^{\circ} \mathrm{C}$ for 8 hours. 
The electrochemical behavior of the PANI-BaMnO 3 nano electrode was analyzed by cyclic voltammetry (CV) and galvanostatic charge-discharge tests at Versa STAT MC electrochemical workstation (Princeton Applied Research). The chemically modified electrode composites consist of $80 \mathrm{wt} \%$ of prepared powders, $10 \mathrm{wt} \%$ of carbon black and $10 \mathrm{wt} \%$ of polyvinylidene difluoride (PVdF). N-methyl-2-pyrrolidine (NMP) was used as a solvent. The mixed slurries were uniformly coated onto a clean surface $(1 \mathrm{~cm} \times 1 \mathrm{~cm})$ of stainless steel plate and heated at $70{ }^{\circ} \mathrm{C}$ overnight to remove the organic solvent. A threeelectrode system was used to analyze the electrochemical properties of the prepared samples. All the measurements were carried out in a $1 \mathrm{M}$ potassium hydroxide electrolyte at room temperature. The prepared electrode was used as working electrode. A platinum foil served as counter electrode, and a saturated calomel electrode (SCE) as reference electrode. Cyclic voltammograms were recorded from 0 to $0.8 \mathrm{~V}$ at scan rates of 10,25 and $50 \mathrm{mVs}^{-1}$. Charge-discharge tests were conducted at different current densities, with cutoff voltage of 0 to 0.8 $\mathrm{V}$.

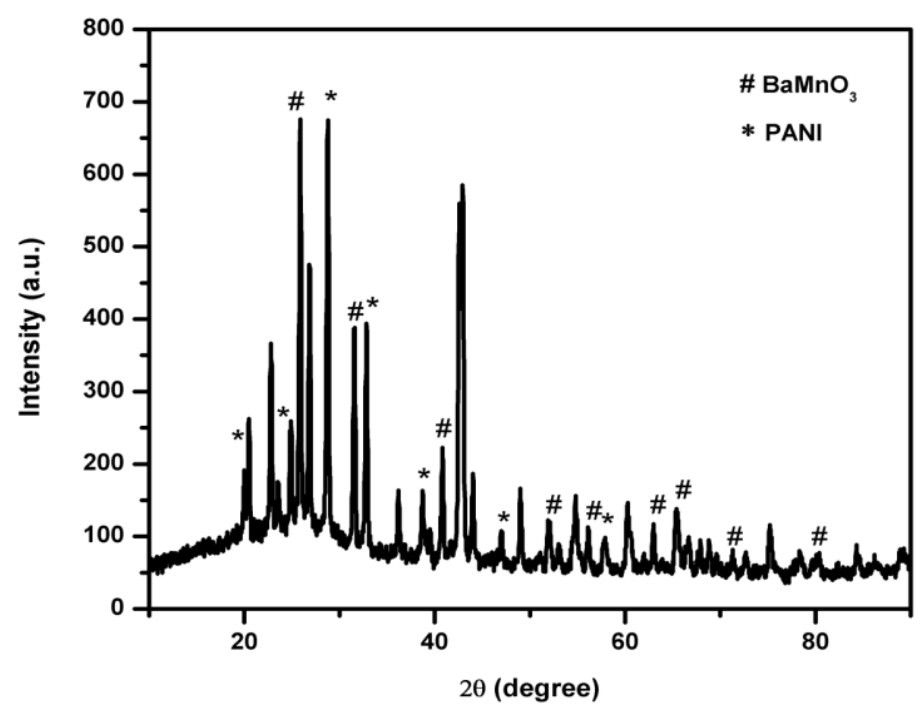

Figure 1. X-ray diffraction patterns of PANI-BaMnO 3 nanohybrid.

\section{Results and discussion}

Fig. 1 shows the XRD patterns of PANI-BaMnO 3 nanohybrid. The sharp and intense peaks that appeared in the diffractogram clearly indicate the high degree of crystallinity of polyaniline by the addition of the $\mathrm{BaMnO}_{3}$ nanocrystalline. Most of the characteristic peaks of the hexagonal $\mathrm{BaMnO}_{3}$ are present in the polymer nanohybrid material, which is consistent with the standard JCPDS values (JCPDS No. 26-0168). The peaks exhibited at 19.7, 23.4, 29.0, 31.3, 32.9, 38.6, 47.1 and $57.8^{\circ}$ are the characteristic peaks of PANI (JCPDS No.53-1717). The crystallinities of PANI and $\mathrm{BaMnO}_{3}$ are complementary to each other. The intensity of diffraction peaks for PANI-BaMnO 3 hybrid is lower than that for pure $\mathrm{BaMnO}_{3}$, which indicates the less crystalline nature of the hybrid material. The diffraction peaks of PANI become intense and narrow, and the intensity of the $\mathrm{BaMnO}_{3}$ peaks is reduced in the hybrid form, in comparison with pure $\mathrm{BaMnO}_{3}$. 
But the intense peaks shown by the hybrid confirm that the amorphous nature of PANI is drastically changed into crystalline form because of the presence of hexagonal $\mathrm{BaMnO}_{3}$ embedded in the PANI matrix. FT-IR spectrum of PANI$\mathrm{BaMnO}_{3}$ nanohybrid is shown in Fig. 2, and it is evident that the spectrum contains contributions from both $\mathrm{BaMnO}_{3}$ and PANI. $\mathrm{C}=\mathrm{N}$ and $\mathrm{C}=\mathrm{C}$ stretching modes for the quinonoid and benzenoid rings occur at $1606 \mathrm{~cm}^{-1}$ and $1402 \mathrm{~cm}^{-1}$, respectively $[21,22]$. The strong band at $1111 \mathrm{~cm}^{-1}$ is the stretching mode of $\mathrm{N}=\mathrm{C}=\mathrm{N}$, and is considered to be a measure of the degree of electron delocalization [23].

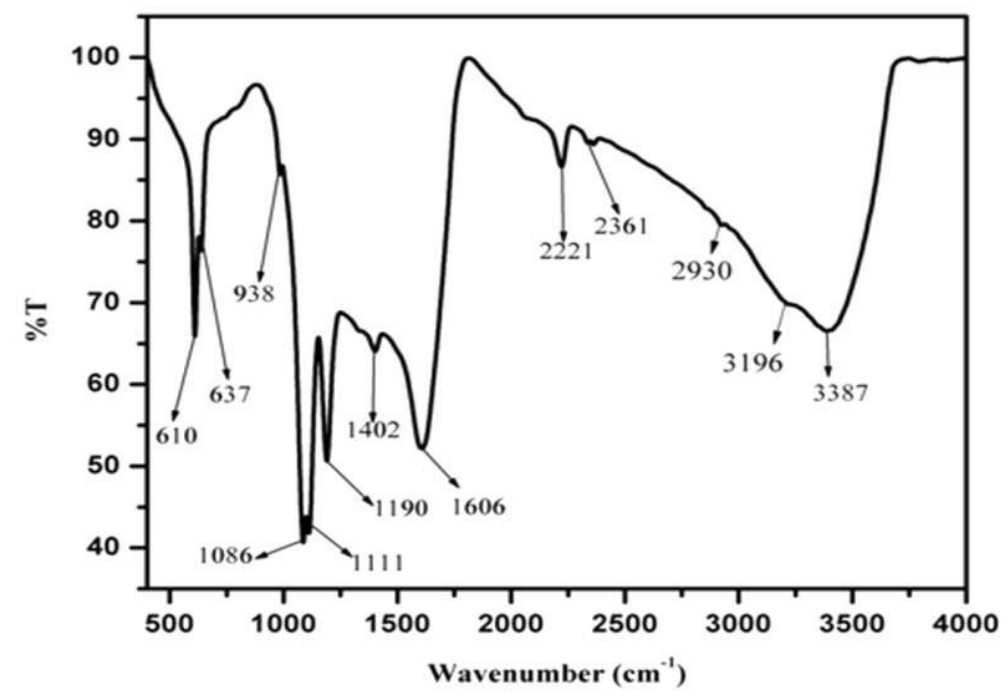

Figure 2. FT-IR spectrum of $\mathrm{PANI}-\mathrm{BaMnO}_{3}$ nanohybrid.

All characteristic bands of PANI are present in the $\mathrm{PANI}-\mathrm{BaMnO}_{3}$ hybrid as well, although the relative intensity of some bands has changed due to the presence of $\mathrm{BaMnO}_{3}$. The strong and intense peak at $610 \mathrm{~cm}^{-1}$ is the characteristic band of metal oxygen bond in $\mathrm{BaMnO}_{3}$. The characteristic band at $3387 \mathrm{~cm}^{-1}$ is attributed to $\mathrm{N}-\mathrm{H}$ stretching mode. However, the intensity of $\mathrm{N}-\mathrm{H}$ stretching of PANI is found to be lower in the PANI- $\mathrm{BaMnO}_{3}$ hybrid, with some shift in band position. This is probably due to the action of hydrogen bonding between the surfaces of the $\mathrm{BaMnO}_{3}$ nanoparticles and the $\mathrm{N}-\mathrm{H}$ groups in PANI. These significant changes suggest that an interfacial interaction exists between PANI and its inorganic $\mathrm{BaMnO}_{3}$ counterpart [24].

The morphology of the PANI- $\mathrm{BaMnO}_{3}$ nanohybrid is depicted in Fig. 3, and it shows homogeneous morphology in the well-defined form of particles ranging from hundreds of nanometers to few microns. Size and shape of the resulting nanostructures primarily depend on the type and amount of surfactants used in the synthesis. In case of a simultaneous use of two different surfactants with varying concentration, it can be observed that changes on surface tension are significantly different from the ones observed when the surfactant alone is used. The mixed surfactant system provides a high level of dispersion of a colloid being formed, to avoid the nanoparticle agglomeration, which results in a formation of nanomaterial with different morphology. 


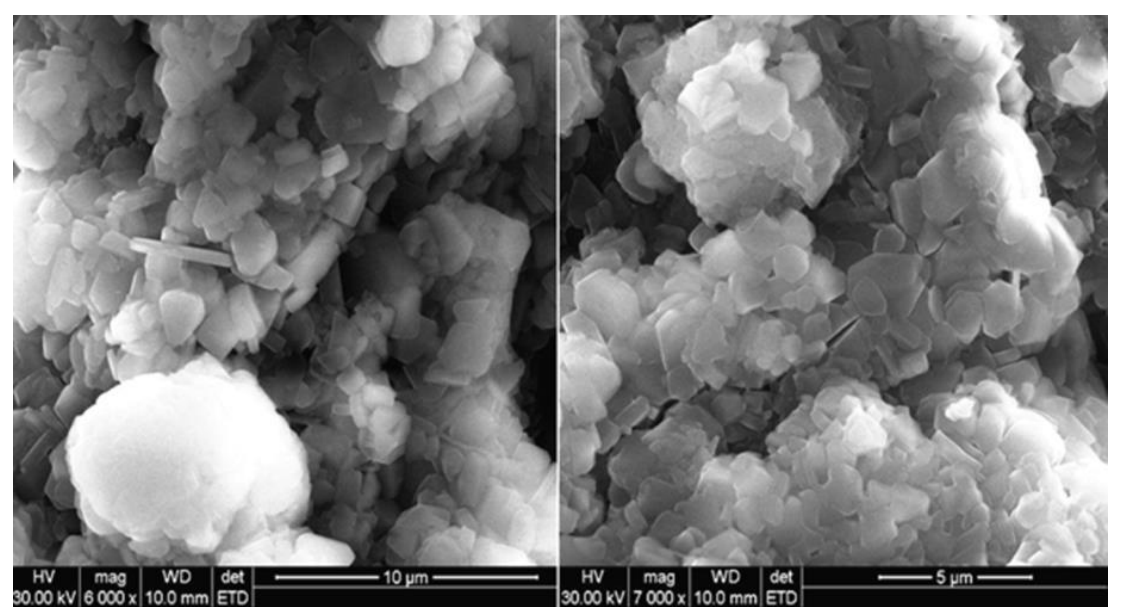

Figure 3. Scanning electron micrographs of $\mathrm{PANI}-\mathrm{BaMnO}_{3}$ nanohybrid.
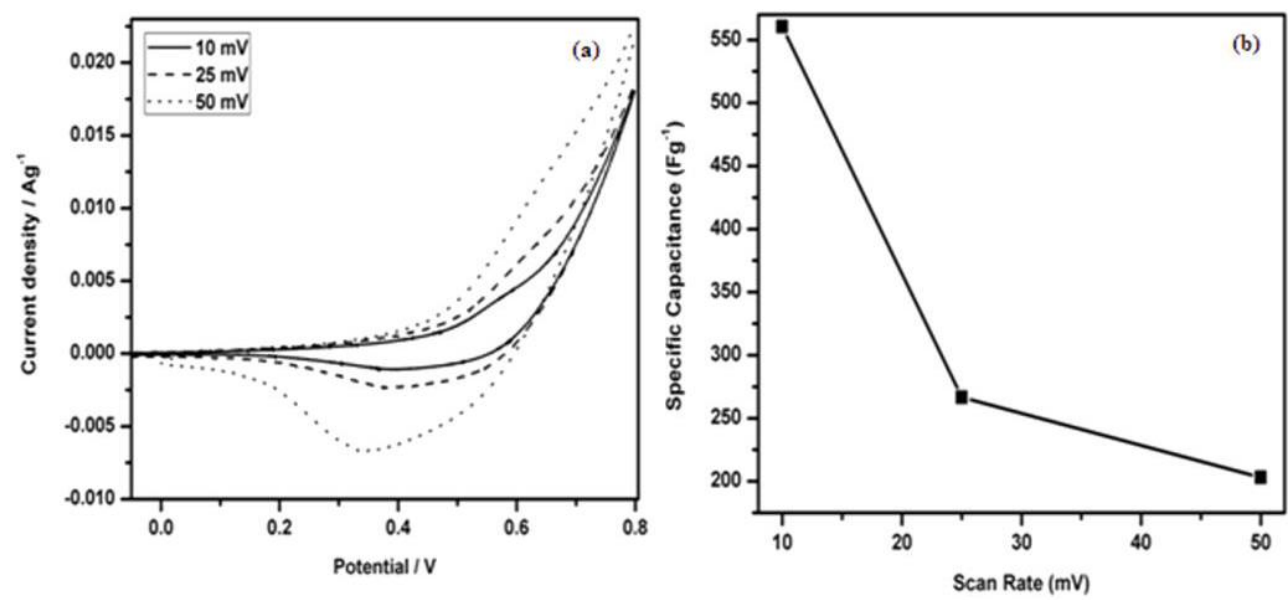

Figure 4. (a) $\mathrm{CV}$ of the $\mathrm{PANI}_{-} \mathrm{BaMnO}_{3}$ nanohybrid electrode in $1 \mathrm{M} \mathrm{KOH}$ solution at different scan rates; (b) scan rate vs. specific capacitance.

A transition metal oxide has multiple positions for doping and tends to bind several nitrogen sites of PANI, forming an inter-chain linkage among several adjacent PANI chains, by coordination. From FESEM images, it is seen that $\mathrm{BaMnO}_{3}$ nanoparticles are uniformly spread on the entire surface of PANI and integrally fixed within PANI chains. Nanosized $\mathrm{BaMnO}_{3}$ is embedded on the PANI matrix; it consists of numerous granular pellets of much refined morphology and shape. This organic-inorganic interaction provides a synergistic effect and prevents the aggregation of PANI during the formation of the hybrid. These pellets are closely and expressly arranged on the surface, and the pores are generated in between the gap of the granular pellets. The porous nature of the hybrid reduces the diffusion resistance of the electrolyte into the electrode matrix and ensures a high reaction rate.

The electrochemical performance of the chemically modified $\mathrm{PANI}-\mathrm{BaMnO}_{3}$ fabricated electrode coupled in $1 \mathrm{M}$ potassium hydroxide was scanned by cyclic voltammogram in the voltage range of $0-0.8 \mathrm{~V}$ at different scan rates, as shown in Fig. 4 (a). 

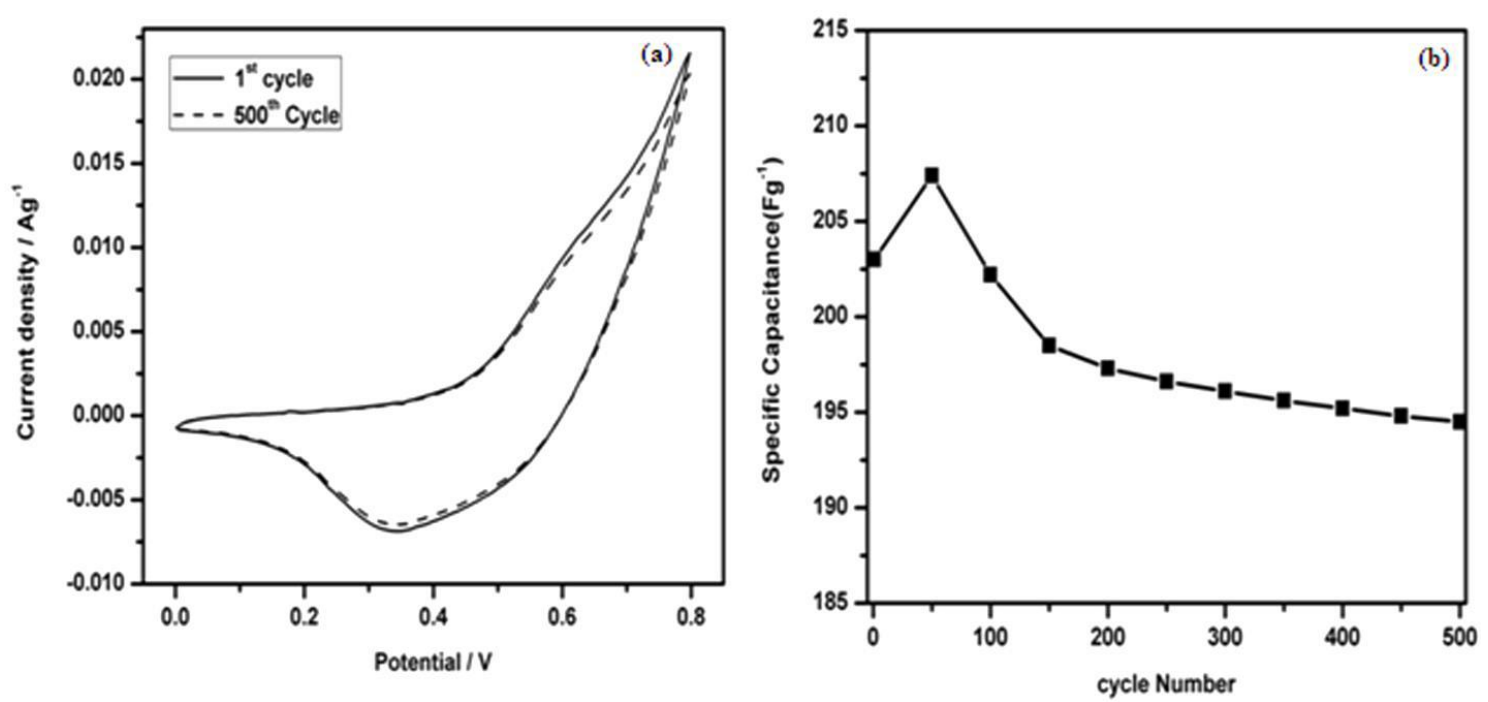

Figure 5. (a) $1^{\text {st }}$ and $500^{\text {th }} \mathrm{CV}$ cycles of PANI-BaMnO 3 nanohybrid electrode in $1 \mathrm{M}$ $\mathrm{KOH}$ solution; (b) cycle number vs. specific capacitance.

The shape of the CV curve reveals that the capacity mainly results from the pseudo capacitance. Supercapacitor parameters such as specific capacitance, discharge capacitance, specific power, specific energy and coulombic efficiency are calculated by using standard equations [25-27]. The specific capacitance values of the capacitor material were calculated at the scan rates of 10, 20 and 50 $\mathrm{mVs}^{-1}$, and were 560.5, 266.5 and $203.0 \mathrm{~F} \mathrm{~g}^{-1}$, respectively. The cyclic voltammogram of PANI-BaMnO 3 at the lower scan rate of $10 \mathrm{mVs}^{-1}$ showed a maximum specific capacitance value, indicating that the electrode material exhibited a redox reaction at both the inner matrix, as well as in the surface, and that it had been sufficiently utilized. The ions completely diffused into the electrode and utilized all the active sites in it at a low current density, resulting in a higher specific capacitance. Similarly, at a higher current density, the ions have a time constraint to utilize all the active sites in the electrode. While increasing the scan rate from 10 to $25 \mathrm{mVs}^{-1}$, the redox peaks fade out slowly, which is mainly attributed to the resistance present in the system, indicating electric polarization. The specific capacitance value dropped to $265 \mathrm{~F} \mathrm{~g} \mathrm{~g}^{-1}$, which is half of the value measured at the scan rate of $10 \mathrm{mVs}^{-1}$. At $50 \mathrm{mVs}^{-1}$ the redox peaks are smoothed and become weak; thus, the specific capacitance value is reduced to $203 \mathrm{~F} \mathrm{~g}^{-1}$. Fig. 4(b) shows the variation of specific capacitance of the PANI$\mathrm{BaMnO}_{3}$ electrode with different scan rates. The reduction of specific capacitance with respect to the increased scan rate indicates that the electrode is charged and discharged at a pseudo constant rate over the complete $\mathrm{CV}$ cycle, which implies very rapid and reversible electrochemical processes. However, even with a very high scan rate at $50 \mathrm{mVs}^{-1}$, the redox peaks are weakened in some extent, but the shape of the CV curve still maintained, and this demonstrates high performance of the supercapacitor. The superior rate capability of PANI-BaMnO 3 hybrid electrodes can be attributed to the highly activated surface and increased electrical conductivity, due to the synergistic contribution from $\mathrm{BaMnO}_{3}$ embedded in the PANI matrix. 
To investigate the cyclic stability of the system, cyclings (500 cycles) carried out at the scan rate of $50 \mathrm{mVs}^{-1}$ are shown in Fig. 5. From the results it is found that the hybrid material can withstand about 500 cycles without a significant decrease in the capacity, and exhibits an excellent cycle profile. The specific capacitance increases from $203 \mathrm{~F} \mathrm{~g}^{-1}$ to $207.4 \mathrm{~F} \mathrm{~g}^{-1}$ over the first 50 cycles, which results from the insufficient activation process of $\mathrm{PANI}-\mathrm{BaMnO}_{3}$. Then, it gradually decreases up to $194.5 \mathrm{~F} \mathrm{~g}^{-1}$ during the following 500 cycles, which is related to the dissolution and detachment of the active material, and to the reduction of active sites in the material surface. The decrease in specific capacitance after 500

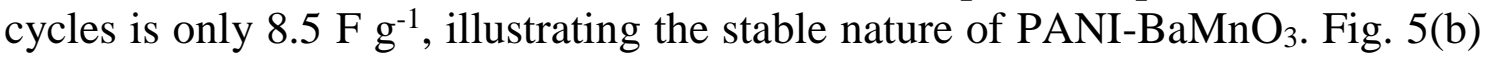
shows the decrease in capacitance with the cycle number of $\mathrm{PANI}-\mathrm{BaMnO}_{3}$ hybrid electrode, and it is observed that the hybrid electrode shows an enhanced stability of $95.82 \%$ over the $500^{\text {th }}$ cycle.
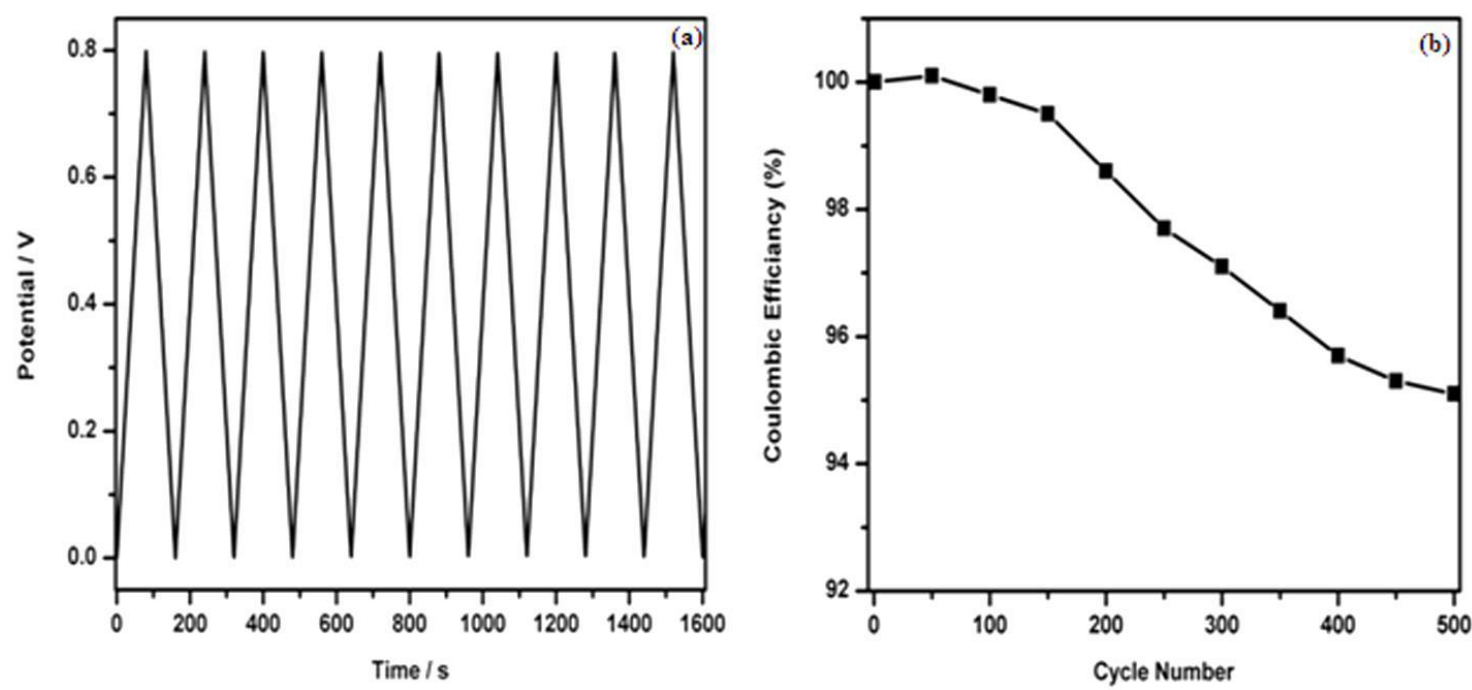

Figure 6. (a) Charge-discharge curves of $\mathrm{PANI}-\mathrm{BaMnO}_{3}$ nanohybrid electrode in $1 \mathrm{M}$ $\mathrm{KOH}$ solution; (b) cycle number vs. coulombic efficiency.

In the charge-discharge profile (Fig.6), the charging curves are exactly symmetrical to the discharging curves. This implies that the $\mathrm{PANI}_{-\mathrm{BaMnO}}$ electrode has good electrochemical reversibility and capacitive characteristics. During the experiments, the amount of charge stored in the capacitor was determined by integrating the current during charge and discharge time. The response of the capacitor shows an ideal linear charge-voltage relationship at lower and higher discharge potential values. From the charge-discharge curve the measured discharge capacitance is $200.3 \mathrm{Fg}^{-1}$, and the capacitance retention is greater than $95 \%$, indicating the efficiency and rate capability of the hybrid. The reason may be that the porous structure of the hybrid material and the synergistic effect provide a relatively easy path for fluid/solid reactions, thus making more redox reactions to occur. The value of specific energy and specific power calculated at $2 \mathrm{mAcm}^{-2}$ discharge current density is $32.01 \mathrm{whkg}^{-1}$ and $400 \mathrm{wkg}^{-1}$, respectively. The coulombic efficiency as a function of the cycle number is shown in Fig. 6 (b). The coulombic efficiency of the capacitor cells was found to be $95.1 \%$ over 500 cycles. 


\section{Conclusions}

$\mathrm{PANI}_{-\mathrm{BaMnO}}$ nanohybrid was successfully prepared by chemical polymerization method. Different techniques such as XRD, FT-IR and FESEM were used to characterize the hybrid material. Doping of nanocrystalline $\mathrm{BaMnO}_{3}$ on the PANI matrix has been shown to be an effective way to increase its capacitive performance, due to the synergistic effect. The prepared hybrid electrode materials exhibit a very high specific capacitance of $560.5 \mathrm{~F} \mathrm{~g} \mathrm{~g}^{-1}$ with excellent cyclic stability. The highest energy and power density was observed as $32.01 \mathrm{Whkg}^{-1}$ and $400 \mathrm{Wkg}^{-1}$, respectively.

\section{References}

1. Wei J, Nagarajan N, Zhitomirsky I. J Mater Process Technol. 2007; 186:356.

2. Jones DJ, Wortham E, J.Roziére J, et al. J Phys Chem Solids. 2004;65:235.

3. Jiang JH, Kucernak A. Electrochim Acta. 2002;47:2381.

4. Ryu KS, Kim KM, Park NG, et al. J Power Sources. 2002;103:305.

5. Sivaraman P, Hande VR, Mishra VS, et al. J Power Sources. 2003;124:351.

6. Jurewicz K, Delpeux S, Bertagna V, et al. Chem Phys Letters. 2001;347:36.

7. Noh KA, Kim DW, Jin CS, et al. J Power Sources. 2003;124:593.

8. Arbizzani C, Gallazzi MC, Mastragostino M, et al. Electrochem Commun. 2001;3:16.

9. $\quad$ Bhat DK, Kumar MS. J Mater Sci. 2007;42:8158.

10. Ohtani A, Abe M, Ezoe M, et al. Synth Met. 1993;55-57:3696.

11. Epstein AJ, Joo J, Kohlman RS, et al. Synth Met. 1994;65:149.

12. Kohlman RS, Min Y, MacDiarmid A, et al. Synth Met. 1995;69:211.

13. Holland ER, Monkman AP. Synth Met. 1995;74:75.

14. Kanatzidis MG, Tonge LM, Marx TJ, et al. J Am Chem Soc. 1987;109:3797.

15. Kanatzidis MG, Wu CC, Marcy HO, et al. J Am Chem Soc. 1989;111:4139.

16. Liu YJ, Degroot DC, Schindler JL, et al. Adv Mater. 1993;5:369.

17. Liu YJ, Degroot DC, Schindler JL, et al. J Chem Soc, Chem Commun. 1993;593.

18. Pokhodenko VD, Koshechko VG, Krylov VA. J Power Sources. 1993;45:15.

19. Pokhodenko VD, Krylov VA. Synth Met. 1991;41-43:533.

20. Shanmugavadivel M, Dhayabaran VV, Subramanian M. J Mat Letters. 2016;181C:335.

21. Jiang J, Ai L, Li LC. J Mater Sci. 2009;44:1024.

22. Zheng L, Wang Y, Wang X, et al. J Power Sources. 2010;195:1747.

23. Mink J, Kristof J, Battisti AD, et al. Surf Sci. 1995;335:252.

24. Li XW, Chen W, Bian CQ, et al. Appl Surf Sci. 2003)

25. Hashmi SA, Latham RJ, Linford RG, et al. Polym Int. 1998;47:28.

26. Prasad KR, Munichandraiah N. J Power Sources. 2002;112:443.

27. Kim IJ, Yang S, Moon SI, et al. J. Power Sources. 2007;164:964. 